University of Nebraska - Lincoln

DigitalCommons@University of Nebraska - Lincoln

2000

\title{
Cross Sections for Singly Differential and Total Ionization of Helium by Electron Impact
}

Yong-Ki Kim

National Institute of Standards and Technology, Gaithersburg, Maryland

Walter R. Johnson

Department of Physics, University of Notre Dame, Notre Dame, Indiana

M. Eugene Rudd

University of Nebraska - Lincoln, erudd@unl.edu

Follow this and additional works at: https://digitalcommons.unl.edu/physicsrudd

Part of the Physics Commons

Kim, Yong-Ki; Johnson, Walter R.; and Rudd, M. Eugene, "Cross Sections for Singly Differential and Total Ionization of Helium by Electron Impact" (2000). M. Eugene Rudd Publications. 26.

https://digitalcommons.unl.edu/physicsrudd/26

This Article is brought to you for free and open access by the Research Papers in Physics and Astronomy at DigitalCommons@University of Nebraska - Lincoln. It has been accepted for inclusion in M. Eugene Rudd Publications by an authorized administrator of DigitalCommons@University of Nebraska - Lincoln. 


\title{
Cross sections for singly differential and total ionization of helium by electron impact
}

\author{
Yong-Ki Kim \\ National Institute of Standards and Technology, Gaithersburg, Maryland 20899-8423 \\ Walter R. Johnson \\ Department of Physics, University of Notre Dame, Notre Dame, Indiana 46556 \\ M. Eugene Rudd \\ Department of Physics and Astronomy, University of Nebraska, Lincoln, Nebraska 68588-0111
}

(Received 23 August 1999; published 10 February 2000)

\begin{abstract}
The binary-encounter dipole (BED) model for electron-impact ionization is applied to helium using the continuum dipole oscillator strength calculated from the relativistic random-phase approximation. The resulting total ionization cross section agrees with available experimental data well within the quoted experimental uncertainties. The singly differential (=energy distribution of ejected electrons) cross section agrees well in shape with available experiments. Because of the remarkable agreement with experiment at all incident and ejected electron energies and the compact analytic form of the cross sections, we propose that the BED cross sections for helium serve as a normalization standard with an accuracy of $\pm 5 \%$ from the threshold to $1 \mathrm{keV}$ in the primary electron energy.
\end{abstract}

PACS number(s): 34.80.Dp

The electron-impact ionization cross section is one of the basic properties of atoms and molecules not only for its intrinsic importance in atomic collision theory, but also for a wide range of applications such as in fusion plasma diagnostics, modeling of semiconductor etching in plasma reactors, radiation effects on materials, and astrophysics.

Helium is ideally suited to serve as a cross section standard. Atomic hydrogen is the only simpler target, but it is difficult to use experimentally. All heavier atoms have the complication of inner shell ionization that poses both theoretical and experimental difficulties. Even in helium double ionization and autoionization from doubly excited states complicate the situation, but together they account for far less than $\pm 5 \%$ in the total ionization cross section. For instance, the cross section for double ionization of helium by electron impact [1] stays below $0.5 \%$ of the cross section for single ionization from the threshold to $1 \mathrm{keV}$ in incident energy.

Numerous theoretical and experimental results on electron-impact ionization of helium have been published since the 1930s. Although most experimental results on the total ionization cross section (TICS) are in excellent agreement with each other [1-4], singly differential cross sections (SDCS) on the energy distribution of ejected electrons are still discordant. The experimental SDCS is commonly obtained by integrating measured angular distribution of ejected electrons, a procedure which entails significant uncertainties in estimating the forward and backward angle cross sections outside the range of direct measurements $[5,6]$.

To emphasize the indistinguishability of the scattered and ejected electrons after an ionizing collision, we follow the standard terminology and designate the faster of the two free electrons as the primary electron and the slower as the secondary electron.

On the theory side, many attempts have also been made, but a comprehensive and accurate description of the ionization process has eluded satisfactory solutions to date [7]. The successful application of the convergent close coupling (CCC) method to electron-impact ionization of helium by Bray and associates [8,9] offers optimism. However, the CCC method is basically for a single-electron target, and requires ever increasing expansion for the coupled states for higher incident electron energies. Although the CCC method can provide detailed collision data in principle, such as the triply differential cross section, the computational procedure for the CCC method is too complicated to provide convenient, analytic expressions for the arbitrary combinations of primary and secondary electron energies needed by experimentalists as a normalization standard for ionization cross sections.

The binary-encounter dipole (BED) model [10] combines a modified form of the Mott cross section [11] with the asymptotic form of the Bethe cross section $[12,13]$ to calculate the SDCS. The resulting SDCS is then integrated over the secondary electron energy to obtain the TICS. The BED model provides the SDCS, $d \sigma / d W$, for a given atomic orbital by using the continuum dipole oscillator strength, $d f / d W$ :

$$
\begin{aligned}
\frac{d \sigma(W, T)}{d W}= & \frac{S}{B(t+u+1)}\left\{\frac{\left(N_{i} / N\right)-2}{t+1}\left(\frac{1}{w+1}+\frac{1}{t-w}\right)\right. \\
& \times\left[2-\left(N_{i} / N\right)\right]\left[\frac{1}{(w+1)^{2}}+\frac{1}{(t-w)^{2}}\right] \\
& \left.+\frac{\ln t}{N(w+1)} \frac{d f(w)}{d w}\right\}
\end{aligned}
$$

where $T$ is the incident electron energy, $W$ is the secondary electron energy, $B$ is the orbital electron binding energy, $U$ $=\left\langle p^{2} / 2 m\right\rangle$ is the orbital electron kinetic energy with the target electron momentum $\boldsymbol{p}$ and the electron mass $m$, $S=4 \pi a_{0}^{2} N R^{2} / B^{2}, a_{0}$ is the Bohr radius, $N$ is the orbital 
electron occupation number, $R$ is the Rydberg energy, $t$ $=T / B, u=U / B, w=W / B$, and

$$
N_{i}=\int_{0}^{\infty} \frac{d f(w)}{d w} d w .
$$

The first term in the curly brackets in Eq. (1), which includes single powers of $w+1$ and $t-w$, comes from the Mott cross section and represents the interference between the direct and exchange interactions, while the second term, which includes squares of $w+1$ and $t-w$, also comes from the Mott cross section and represents the direct and exchange interactions. The logarithmic term in the brackets is the leading term of the Bethe cross section.

The factor $2-N_{i} / N$ results from the requirement that the asymptotic limits of both the TICS and the stopping cross section approach the known asymptotic limits of the Bethe theory $[10,12]$. This requirement eliminates the need for an arbitrary/empirical parameter often used in previous attempts to combine the binary-encounter theory and the Bethe cross section [14].

The TICS is obtained by integrating Eq. (1) over $w$ between $w=0$ and $(t-1) / 2$ :

$$
\sigma_{i}(t)=\frac{S}{t+u+1}\left[D(t) \ln t+\left(2-\frac{N_{i}}{N}\right)\left(\frac{t-1}{t}-\frac{\ln t}{t+1}\right)\right],
$$

where

$$
D(t) \equiv N^{-1} \int_{0}^{(t-1) / 2} \frac{1}{w+1} \frac{d f(w)}{d w} d w .
$$

Note that the Mott cross section part of Eq. (1) is symmetric with respect to the energy of the primary and secondary electrons after the collision, and hence the upper limit of integration in Eqs. (3) and (4) is chosen to avoid double counting.

The continuum oscillator strength, $d f / d w$, was calculated using the relativistic random-phase approximation (RRPA) [15], though it is not necessary to introduce relativity for helium. It should be noted that RRPA oscillator strengths for dipole excitations of a closed-shell $\mathrm{N}$-electron atom automatically satisfy the Thomas-Reiche-Kuhn sum rule,

$$
\sum_{i} f_{i}+\int_{0}^{\infty} \frac{d f(w)}{d w} d w=N
$$

where $i$ ranges over all odd-parity $J=1$ bound states. Moreover, the gauge-independence of RRPA amplitudes insures that length-form and velocity-form oscillator strengths are identical.

To be able to integrate $d f / d w$ to an arbitrary upper limit, we fitted the $d f / d w$ calculated from the RRPA to a four-term power series:

$$
d f / d w=a y^{3}+b y^{4}+c y^{5}+d y^{6},
$$

where $y=B / E=B /(W+B), \quad$ and $\quad a=8.24012, \quad b$ $=-10.4769, c=3.96496$, and $d=-0.0445976$. To match the experimental threshold, we use $B=24.587 \mathrm{eV}$, while we use a theoretical value of $U=39.51 \mathrm{eV}$.

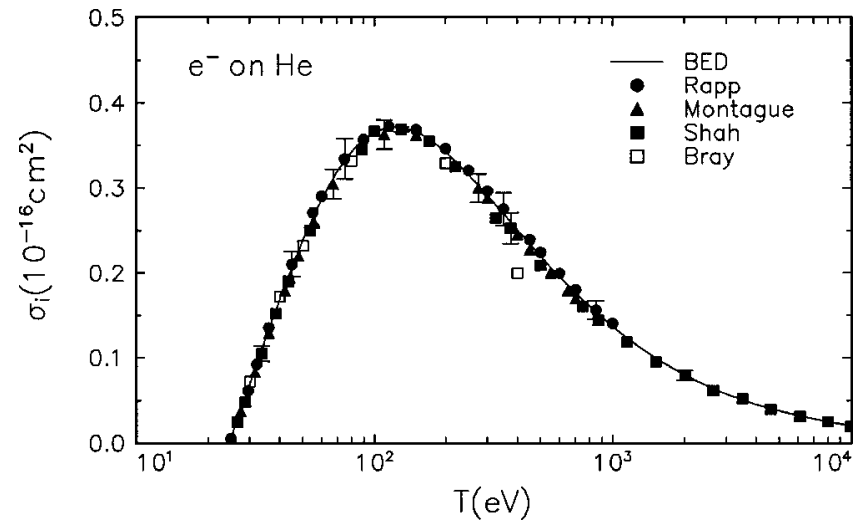

FIG. 1. Total cross section for ionization of He by electron impact. The abscissa is the incident electron energy $T$ in eV. Solid line, BED cross section; filled circles, experimental data by Rapp and Englander-Golden [3]; filled triangles, data by Montague et al. [4]; filled squares, data by Shah et al. [1]; open squares, CCC theory by Fursa and Bray [8].

With Eqs. (1)-(6) and the fitting constants above, one can generate $d \sigma / d W$ and $\sigma_{i}$ for arbitrary $T$ and $W$, even for $T$ near the ionization threshold and for $W \sim 0$, two regions which pose severe difficulties to experiment.

In Fig. 1, we compare $\sigma_{i}$ from the BED model to three sets of experimental data, which agree well among themselves. Our BED results fall well within the uncertainties of the experimental data, which range between $\pm 5 \%$ and $\pm 7 \%$, indicating that the TICS from the BED model presented here can be used as a normalization standard with a maximum uncertainty of $\pm 5 \%$. The CCC cross section [8] at $T<100 \mathrm{eV}$ is also in excellent agreement with the experiments, though the CCC cross section falls below the experiments at higher $T$, indicating the need for extending the basis set.

The SDCS at $T=100 \mathrm{eV}$ is compared in Fig. 2 to experimental data, which were obtained by integrating doubly differential cross sections (DDCS), or the angular distribution of secondary electrons. The integration of DDCS requires

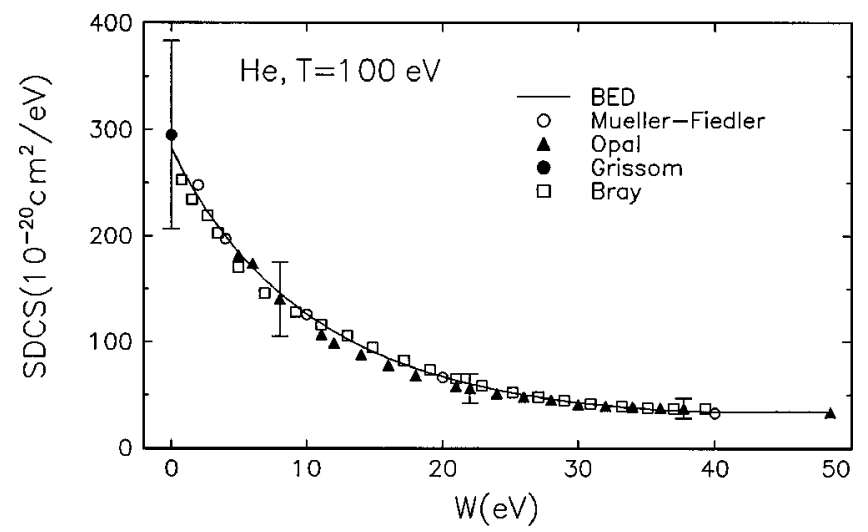

FIG. 2. Singly differential cross section of He at $T=100 \mathrm{eV}$. Solid line, BED cross section; open circles, experimental data by Müller-Fiedler et al. [6]; filled triangles, data by Opal et al. [5]; filled circle, data by Grissom et al. [17]; open squares, CCC theory by Bray and Fursa [9]. 
extrapolations of experimental data to forward and backward angles. For fast secondary electrons, the angular distribution is sharply peaked around the binary collision peak, and the accuracy of the integration of such a peak will depend on the size of the angular steps used for observation. In spite of these experimental difficulties, the agreement between the BED model and experimental data on SDCS shown in Fig. 2 is remarkable.

A more informative way to compare SDCS is to use the Platzman plot [16], which is the ratio of SDCS to the Rutherford cross section for one electron:

$$
Y \equiv \frac{d \sigma / d W}{4 \pi a_{0}^{2} R^{2} / T E^{2}}
$$

as a function of $R / E$. Note that the simple relationship, $E$ $=W+B$, holds only for targets with a single orbital, such as atomic hydrogen, helium, and molecular hydrogen.

In the Platzman plot, the ordinate, $Y$, indicates the effective number of electrons being ionized-as in the concept of dipole oscillator strength-while the area under the curve between $W=0$ and $W_{\max }=(T-B) / 2$ is $\left(T / 4 \pi a_{0}^{2} R\right) \sigma_{i}$. In other words, the Platzman plot compares not only the shape but also the normalization of SDCS.

Comparison of theoretical and experimental SDCS at $T$ $=500 \mathrm{eV}$ is shown as a Platzman plot in Fig. 3, which clearly demonstrates that the shapes of the two sets of experimental data are similar, but their magnitudes must be renormalized to match the known TICS. Comparisons of SDCS at other primary electron energies exhibit the same trend, and we are confident that the shape and magnitude obtained from Eq. (1) are trustworthy, particularly for $T \leqslant 1$ $\mathrm{keV}$.

In both Figs. 2 and 3 we included the experimental data by Grissom et al. [17], who measured $d \sigma / d W$ at $W=0$. This is a unique experiment which uses a potential trap and provides SDCS at $W=0$, which is an ejected electron energy that is very difficult to measure by conventional methods.

Although we have presented a successful application of the BED model, the model has some inherent limitations owing to its relatively simple origin. The Mott cross section uses Coulomb wave functions for the primary and secondary electrons. The Bethe cross section uses plane waves for the primary electron, while realistic wave functions should be used for the initial and final target states to calculate $d f / d w$. Therefore, these two theories, though well understood, should be combined with care.

Furthermore, the exchange effect between the primary and secondary electrons is fully accounted for in the Mott cross section, while the Bethe cross section ignores it. The electron correlation included in the RRPA is between the two bound electrons of helium, but not between the primary and

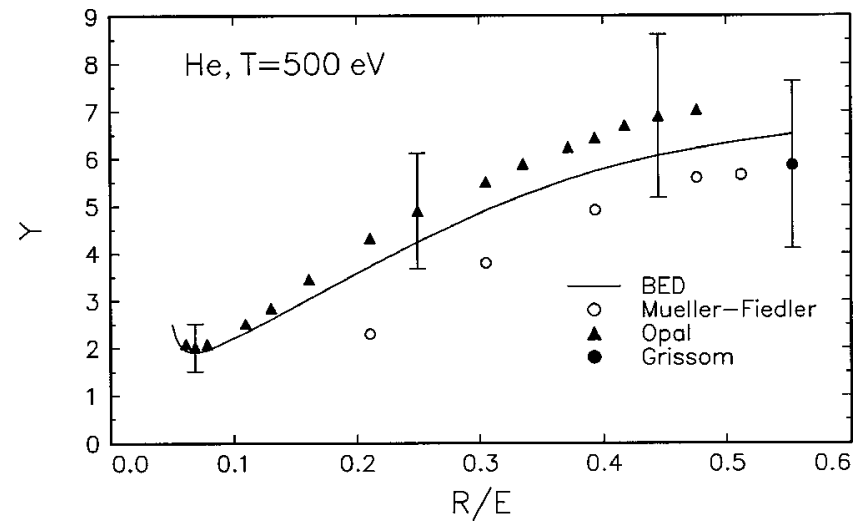

FIG. 3. Platzman plot of $\mathrm{He}$ at $T=500 \mathrm{eV}$. Solid line, BED cross section; open circles, experimental data by Müller-Fiedler $e t$ al. [6]; filled triangles, data by Opal et al. [5]; filled circle, data by Grissom et al. [17].

secondary electrons. Fortunately, the latter exchange effect diminishes at high $T$, where the Bethe cross section begins to dominate. The fact that the TICS based on the BED model agrees so well with experiment for helium is a strong indication that at low $T$ the dipole interaction is less important than the non-dipole interaction represented by the Mott cross section.

Our preliminary results on the application of the BED model with $d f / d w$ from the RRPA to neon yields a level of agreement in the TICS between $T=50$ and $500 \mathrm{eV}$ similar to that for helium. Details of the BED cross sections of helium and neon with $d f / d w$ from the RRPA will be reported later. Examples of BED cross sections and a simplified version of the theory-the binary-encounter-Bethe (BEB) cross sections-for some atoms and dozens of molecules as well as on-line computation of SDCS and TICS of atomic hydrogen, helium, and molecular hydrogen for arbitrary $T$ and $W$ are available on the Internet [18].

We conclude that the energy distribution and the total ionization cross section of helium given by the BED model, Eqs. (1)-(6), with the $d f / d w$ calculated from the RRPA can be used as a normalization standard for electron-impact ionization experiments on atoms and molecules. The theoretical data are expected to be accurate to $\pm 5 \%$ based on the comparison with available experimental data, and where applicable, with the CCC theory.

The work at NIST was supported in part by the Office of Fusion Energy Sciences, U. S. Department of Energy, and by the Advanced Technology Program of NIST. The work at the University of Notre Dame was supported by the National Science Foundation under Grant No. PHY-99-70666.
[1] M. B. Shah, D. S. Elliot, P. McCallion, and H. B. Gilbody, J. Phys. B 21, 2751 (1988).

[2] P. T. Smith, Phys. Rev. 36, 1293 (1930).

[3] D. Rapp and P. Englander-Golden, J. Chem. Phys. 43, 1464
(1965).

[4] R. G. Montague, M. F. A. Harrison, and A. C. H. Smith, J. Phys. B 17, 3295 (1984).

[5] C. B. Opal, E. E. Beaty, and W. K. Peterson, At. Data 4, 209 
(1972).

[6] R. Müller-Fiedler, K. Jung, and H. Ehrhardt, J. Phys. B 19, 1211 (1986).

[7] See, for example, S. M. Younger, in Electron-Impact Ionization, edited by T. D. Märk and G. H. Dunn (Springer-Verlag, Wien, 1985), Chap. 1.

[8] D. V. Fursa and I. Bray, Phys. Rev. A 52, 1279 (1995); J. Phys. B 30, 5895 (1997), and references therein.

[9] I. Bray and D. V. Fursa, Phys. Rev. Lett. 76, 2674 (1996); I. Bray and D. V. Fursa, Phys. Rev. A 54, 2991 (1996), and references therein.

[10] Y.-K. Kim and M. E. Rudd, Phys. Rev. A 50, 3954 (1994).
[11] N. F. Mott, Proc. R. Soc. London, Ser. A 126, 259 (1930).

[12] H. Bethe, Ann. Phys. (Leipzig) 5, 325 (1930).

[13] M. Inokuti, Rev. Mod. Phys. 43, 297 (1971).

[14] See, for instance, S. P. Khare and W. J. Meath, J. Phys. B 20, 2101 (1987).

[15] W. R. Johnson and C. D. Lin, Phys. Rev. A 20, 964 (1979); W. R. Johnson and K. T. Cheng, ibid. 20, 978 (1979).

[16] Y.-K. Kim, Radiat. Res. 61, 21 (1975); 64, 96 (1975); 64, 205 (1975).

[17] J. T. Grissom, R. N. Compton, and W. R. Garrett, Phys. Rev. A 6, 977 (1972).

[18] http://physics.nist.gov/ionxsec 